\title{
Exercise prescription and thrombogenesis
}

\author{
Jong-Shyan Wang* \\ Graduate Institute of Rehabilitation Science and Center for Gerontological Research, Chang Gung University, \\ 259 Wen-Hwa 1st Road, Kwei-Shan, Tao-Yuan, 333, Taiwan
}

Received 28 August 2005; accepted 14 July 2006

(C) 2006 National Science Council, Taipei

Key words: acute exercise, coagulation, deconditioning, exercise training, fibrinolysis, platelets

\begin{abstract}
Summary
Lifestyle habits, such as exercise, may significantly influence risk of major vascular thrombotic events. The risk of primary cardiac arrest has been shown to transiently increase during vigorous exercise, whereas regular moderate-intensity exercise is associated with an overall reduced risk of cardiovascular diseases. What are the mechanisms underlying these paradoxical effects of vigorous exercise versus exercise training on thrombotic modification? This review analyzes research regarding effects and their underlying mechanisms of acute exercise, endurance training, and deconditioning on platelets, coagulation, and fibrinolysis. Evidence suggests that (i) light, acute exercise ( $\leq 49 \% \mathrm{VO}_{2}$ max $)$ does not affect platelet reactivity and coagulation and increases fibrinolytic activity; (ii) moderate, acute exercise $\left(50 \sim 74 \% \mathrm{VO}_{2 \max }\right)$ suppresses platelet reactivity and enhances fibrinolysis, which remains unchanged in the coagulation system; and, (iii) strenuous, acute exercise $\left(\geq 75 \% \mathrm{VO}_{2 \max }\right)$ enhances both platelet reactivity and coagulation, simultaneously promoting fibrinolytic activity. Therefore, moderate exercise is likely a safe and effective exercise dosage for minimizing risk of cardiovascular diseases by inducing beneficial anti-thrombotic changes. Moreover, moderate-intensity exercise training reduces platelet reactivity and enhances fibrinolysis at rest, also attenuating enhanced platelet reactivity and augmenting hyper-fibrinolytic activity during strenuous exercise. However, these favorable effects of exercise training on thrombotic modification return to a pretraining state after a period of deconditioning. These findings can aid in determining appropriate exercise regimes to prevent early thrombotic events and further hinder the cardiovascular disease progression.
\end{abstract}

\section{Benefits and risks associated with exercise in cardiovascular diseases}

Increased physical activity [1] and cardiorespiratory fitness [2] are associated with a reduced risk of fatality from coronary heart disease, as well as other causes. Moreover, increased studies have demonstrated that regular moderate-intensity physical activity is associated with health benefits, even when aerobic fitness (e.g., maximal oxygen uptake, $\left.\mathrm{VO}_{2 \max }\right)$ remains unchanged [3]. For sedentary

*To whom correspondence should be addressed. Fax: + 886-32118700; E-mail: s5492@mail.cgu.edu.tw persons at risk for cardiovascular disease, adopting a moderately active lifestyle can induce important health benefits, such as reducing blood pressure [4], prothrombotic factors [5], and lipid peroxidation [6], increasing high-density lipoprotein levels [6], fibrinolytic activity [7], and vasodilatation [8], which represent more attainable goals than high $\mathrm{VO}_{2 \max }$.

Conversely, clinical investigations conferred that strenuous, acute exercise can enhance risk of major vascular thrombotic events and transiently increase incidence of primary cardiac arrest $[9,10]$. The incidence of cardiovascular complications during exercise is substantially greater for those with cardiovascular disease than for healthy 
adults. In the largest study to date, comprising over 1 million exercise tests, no fatal and nonfatal complications occurred in athletes, whereas coronary patients had morbidity and mortality rates of 1.4 and 0.2 per 10,000 tests, respectively [11]. For patients with coronary artery disease, relative risk of cardiac arrest during vigorous exercise was over 100 -fold greater than what might be expected to occur spontaneously [12]. Moreover, the relative risk of acute myocardial ischemia in these patients was also 2-6 times higher during strenuous physical exertion ( $>6$ METs) compared with during mild-to moderate-intensity activities; however, the risk varies greatly depending on the patient's usual frequency of physical activity [13, 14]. Therefore, physical exercise seems to be able to protect individuals against cardiovascular disease on the one hand and to provoke sudden cardiac death on the other hand, and according to reactions reliant on the intensity of exercise.

Exercise prescriptions aim to enhance physical fitness, promote health by reducing risk factors for chronic diseases, such as cardiovascular disease, and ensure safety during exercise. The principal components of a systematic, individual exercise prescription comprise an appropriate mode, intensity, duration, frequency, and progression of exercise [15]. According to recommendations by the Center for Disease Control and Prevention (CDC) and the American College of Sports Medicine (ACSM), exercise intensity is the primary factor when prescribing an exercise regimen to protect individuals against cardiovascular disease [3]. The optimal exercise prescription for an individual is based on an objective evaluation of the individual's response to exercise, including heart rate (HR), rating of perceived exertion (RPE), $\mathrm{VO}_{2 \max }$, and metabolic equivalents
(MET) during a graded exercise test [15]. Table 1 presents the classifications for activity intensity and recommended quantity and quality of exercise for developing and maintaining health-related physical fitness in healthy adults based on ACSM guidelines for exercise prescriptions [15].

This review summarizes and evaluates research into the effects of acute exercise, endurance training, and deconditioning/disability on thrombotic modification, particularly on platelet reactivity, coagulation, and fibrinolysis, and their underlying mechanisms. By elucidating the relationship between physical activity and thrombogenesis, this study attempts to provide a strategy for developing suitable exercise regimens that prevent early thrombotic events, further retarding the progression of cardiovascular disease.

\section{Exercise and changes in platelet reactivity}

\section{Effects of acute exercise}

Pathological and clinical studies have indicated that platelets are critical to pathogenesis and progression of cardiovascular disease [16]. Various studies identified increased platelet counts of $18-80 \%$ immediately after treadmill or bicycle exercising [17]. Moreover, the degree to which platelet counts were enhanced by acute exercise was related to exercise severity [18]. Increased platelet count induced by acute exercise can be attributed to the release of platelets from the spleen's vascular beds, bone marrow, and intravascular pool of pulmonary circulation [17]. Recent studies also demonstrated that platelet adhesion and aggregation by physical shear forces and chemical agonists was affected by acute

Table 1. Classification of physical activity intensity.

\begin{tabular}{lcccc}
\hline Intensity & $\% \mathrm{VO}_{2 \max }$ & $\% \mathrm{HR}_{\max }$ & Category RPE scale & MET \\
\hline Very light & $<30$ & $<35$ & $<10$ & $<3$ \\
Light & $30-49$ & $35-59$ & $10-11$ & $3-4$ \\
Moderate & $50-74$ & $60-79$ & $12-13$ & $5-6$ \\
Strenuous & $75-84$ & $80-89$ & $14-16$ & $7-8$ \\
Very strenuous & $>85$ & $>90$ & $>16$ & $>8$ \\
\hline
\end{tabular}

$\mathrm{VO}_{2 \max }=$ maximal oxygen consumption; $\mathrm{HR}_{\max }=$ maximal heart rate; $\mathrm{PRE}=$ rating of perceived exertion; MET $=$ metabolic equivalents. (1 MET is considered equivalents to a $\mathrm{VO}_{2}$ of $3.5 \mathrm{ml} / \mathrm{min} / \mathrm{kg}$ and represents an energy expenditure of approximately $1.2 \mathrm{kcal} / \mathrm{min}$ for a $70 \mathrm{~kg}$ person). 
exercise in intensity manner [18-25]. Additionally, physically active and sedentary subjects respond differently to the same exercise regimen. According to previous studies by the author, strenuous exercise (exercise up to $\mathrm{VO}_{2 \max }$ ) increased platelet adhesiveness on fibrinogen-coated surface and ADP-induced aggregation; whereas moderate exercise $\left(60 \% \quad V_{2} O_{2 \max }\right.$ for $\left.30 \mathrm{~min}\right)$ desensitized the platelet reactivity in healthy males and male patients with stable angina [18]. Exercise effects were also more pronounced in sedentary than in the active males. Kestin et al. also demonstrated that strenuous exercise promoted platelet hyperactivity in sedentary men, as assayed by the presence of activation-associated surface antigens on platelet. However, for physically active subjects, exercise did not produce consistent changes in platelet activity [26].

Notably, platelet activation induced by shear forces in a stenosed coronary artery is one of the mechanisms of coronary thrombosis [27]. Shear stress levels in stenotic arteries reach from 60 to 3300 dynes $/ \mathrm{cm}^{2}$ [28]. By simulating flow conditions in stenotic arteries using a rotational viscometer, measurement results from previous studies by the authors demonstrated that strenuous exercise ( $80 \%$ $\mathrm{VO}_{2 \max }$ for $40 \mathrm{~min}$ ) enhanced platelet aggregation under high shear stress (100 dyne $\left./ \mathrm{cm}^{2}\right)$, possibly by improving the ability of von Willbrand factor (vWF) binding to platelets and, subsequently, activating glycoprotein IIb/IIIa (GPIIb/IIIa) and P-selectin expression on platelets [29]. Furthermore, a clinical investigation also displayed that shearinduced platelet aggregation in patients with effort angina enhanced after strenuous treadmill exercise [30]. Conversely, moderate exercise $\left(60 \% \mathrm{VO}_{2 \max }\right.$ for $40 \mathrm{~min}$ ) suppressed agonist-and shear-induced platelet aggregation via reduced vWF binding to platelets and down-regulation of platelets GPIIb/ IIIa activation and P-selectin expression [31].

Both $\beta$-thromboglobulin ( $\beta$-TG) and platelet factor 4 (PF4) are released from platelet $\alpha$ granules during in vivo platelet activation. Although both $\beta$-TG and PF4 are present in similar amounts in platelets and are released in similar quantities, plasma levels of $\beta \mathrm{T}-\mathrm{G}$ exceed those of PF4 due to rapid removal of PF4 in circulation [32]. Additionally, artifacts resulting from blood sampling and handling can induce elevated $\beta$-TG and PF4 levels in plasma [32]. Although investigations showed that $\beta$-TG and PF4 concentrations increased following acute exercise [33, 34], other studies did not identify significant changes in the ratio of $\beta$-TG to PF4 after exercise [18]. Therefore, this finding suggests that platelet secretion in vivo was not directly activated by exercise.

What are the mechanisms underlying the opposing effects of strenuous and moderate exercise on platelet reactivity? Augmentation of shearinduced platelet aggregation during strenuous exercise is likely related to increased endogenous release of epinephrine. Strenuous exercise promotes the release of epinephrine [35], which can induce the release of $\mathrm{vWF}$ from endothelial cells [36] and enhance shear-induced platelet aggregation via activating platelet $\alpha_{2}$-adrenergic receptors [37]. Epinephrine can synergize with hear stress to induce platelet aggregation, and this synergistic response depends on both $\mathrm{vWF-GPIb}$ interaction and functional GPIIb/IIIb complexes [38]. An early study indicated that strenuous exercise improved performance of platelet $\alpha_{2}$-adrenergic receptors by increasing endogenous release of epinephrine, thereby promoting activation of fibrinogen receptors (as GP IIb/IIIa complex) and fibrinogen binding to platelet fibrinogen receptors [20]. Additionally, strenuous exercise also enhanced $\alpha_{2}$-adrenergic receptor-mediated platelet $\left[\mathrm{Ca}^{2+}\right]_{i}$ elevation, suggesting that exocytosis of $\alpha$-granules and subsequent translocation of P-selectin in platelet may be increased by strenuous exercise $[20,29]$. Therefore, the endogenous release of epinephrine and activation of platelet $\alpha_{2}$-adrenergic receptor induced by strenuous exercise can be partially explained by enhanced shearinduced platelet aggregation via the vWF-GPIb interaction, GPIIb/IIIa activation, and P-selectin expression during strenuous exercise. Compared with strenuous exercise, moderate exercise induced lower levels of catecholamines and a higher release of nitric oxide (NO) from vascular endothelial cells [23]. Nitric oxide inhibits thrombus formation under high shear flow and attenuates agonistinduced upregulation of P-selectin and the GPIIb/ IIIa complex by negatively regulating the cGMP in platelets $[39,40]$. By increasing NO release, moderate exercise can decrease the performance of adhesion molecules on platelets, thereby reducing platelet activation induced by shear stress.

To determine the effects of acute exercise on platelet functional behavior in women, the authors performed an experimentation using female subjects 
without knowing at which point of their menstrual cycle when the exercise was executed; measurement results showed that both strenuous exercise and moderate exercise did not affect platelet reactivity. When based on menstrual cycle phases, platelet adhesion and aggregation were enhanced by strenuous exercise and suppressed by moderate exercise in the mid-follicular menstrual phase and no platelet functions were impacted by these two exercises during the mid-luteal menstrual phase [22]. Moreover, these exercise-induced platelet functional changes were mediated by NO [22]. Epidemiological investigations have demonstrated that pre-menopausal women have a low incidence of cardiovascular disease [41]. The unresponsiveness of platelets to acute exercise in the luteal menstrual phase can partially explain why premenopausal women have a lower incidence of cardiovascular diseases than do men.

Although strenuous exercise enhances platelet reactivity, whether it has a negative feedback pathway acting against this platelet-related thrombotic risk evoked by exercise is unclear. Findings from a recent study for to the exercise effects on platelet-leukocyte interaction may provide a novel clue explaining the negative feedback pathway for exercise-induced platelet reactivity [21]. Reciprocal modulation of platelet and polymorphonuclear leukocyte $(\mathrm{PMN})$ activities is important to thrombotic pathogenesis [42]. Platelet activation under pathological vascular conditions, such as fissured atheromas or growing thrombi, can stimulate PMN activity, amplifying the thrombotic process by activating platelets [43]. However, PMN also exerts an anti-thrombotic influence by inhibiting platelet activation, such as PMN-derived NO [44, 45], whereas platelet activation also counterbalances the harmful effects of excessive PMN stimulation by increasing substances, such as soluble P-selectin (sP-selectin), released upon platelet activation [46]. Some studies demonstrated that platelet-leukocyte aggregation enhanced after exercise gradually increased in intensity until exhaustion under a lack of shear flow control or static conditions [47]. More recently, Wang et al. further demonstrated that although sustained strenuous exercise $\left(80 \% \mathrm{VO}_{2 \max }\right.$ for $\left.40 \mathrm{~min}\right)$ increased the number of platelets binding to PMNs under a flow condition mimicking a venous circuit (5 dyne/ $\mathrm{cm}^{2}$ ), platelet-PMN interactions under high shear stress mimicking normal arterial (10-30 dyne $\left./ \mathrm{cm}^{2}\right)$ or stenotic arterial ( $\geq 80 \mathrm{dyne} / \mathrm{cm}^{2}$ ) flow were suppressed. Moreover, strenuous exercise was also associated with increased PMN-derived NO metabolite levels and PMN-dependent inhibition of platelet activation [21]. Therefore, the paradoxical effects of shear-mediated platelet-PMN interaction induced by strenuous exercise may be the negative feedback pathway against the risk of exercise-induced platelet-related thrombosis. On the other hand, moderate exercise increased plasma and PMN-derived NO levels, enhanced PMN-dependent inhibition of platelet activation, and suppressed shear-induced platelet activation and subsequent adhesion of PMNs to platelets deposited at sites of vascular injury under flow [31], thereby reducing risk of vascular thrombosis and inflammation.

\section{Effects of exercise training and physical deconditioning}

The beneficial effects of exercise training are mediated by several factors, including physiological improvement to the advocated haemostatic equilibrium, such as suppressed pro-thrombogenetic factors and enhanced anti-thromobogenetic factors. Previous studies have suggested that risk of primary cardiac arrest increases transiently during vigorous exercise [14], whereas regular physical exercise is associated with overall reduced risk of primary cardiac arrest [13]. An animal study showed that exercise training reduced the tendency toward thrombosis induced by laser injury to blood vessels [48]. Some studies have identified that a moderateintensity exercise training $\left(60 \% \mathrm{VO}_{2 \max }\right.$ for $30 \mathrm{~min}$ per day, 5 days/week for 8 weeks) reduced resting and strenuous exercise-induced platelet aggregation and platelet adhesion under shear flow in men [19], and in women in a mid-follicular menstrual phase [23], which were accompanied by decreased vWF binding to platelets and expression of P-selectin on platelets [49]. Several mechanisms potentially explain why exercise training reduced the extent of shear-induced platelet activation. First, exercise training decreases resting and strenuous exerciseinduced plasma catecholamine levels $[35,50]$ and down-regulates platelet $\alpha_{2}$-adrenergic receptor performance [51], thereby reducing vWF-platelet interaction and platelet $\mathrm{P}$-selectin expression under shear flow. Second, exercise training enhances substantial NO release from platelets and the endothelium, thus 
increasing platelet cGMP level and reducing the capacity of adhered platelets to withstand shear stress [23]. Additionally, regular moderate-intensity exercise reduces the susceptibility of low-density lipoprotein (LDL) to undergo oxidation [52], and suppresses the oxidized LDL-promoted capacity of adhered platelets to withstand shear stress via enhancing anti-oxidative activity [53-55]. Therefore, exercise training suppresses oxidative modification of lipid profiles by increasing antioxidant release or production to attenuate oxidative stresspromoted platelet activation under the pathological shear flow condition.

Suppressed resting platelet reactivity induced by exercise training, which may, in turn, reduce risk of thrombotic events. This mechanism may explain, at least partially, why regular exercise protects against cardiovascular diseases. Moreover, the platelet activity enhanced by strenuous exercise diminished after exercise training. This diminishment may reduce risk of primary cardiac arrest during vigorous exercise in trained subjects.

Conversely, reduced daily activity, such as in sedentary lifestyles, physical deconditioning, and limb immobilization and paralysis (e.g., spinal cord injury (SCI)), decreases cardiopulmonary fitness and increases risk for major thrombotic events, leading to further cardiovascular and haemostatic disorders. In physical deconditioning, studies have demonstrated that although resting and strenuous exercise-induced platelet adhesiveness and aggregability were reduced by 8 weeks of exercise training, deconditioning for $4-8$ weeks returned platelet reactivity to a pre-training state [19, 23, 49]. Under physical disability, individuals with SCI had more extensive basal and arm crank exercise-induced platelet activation and $\mathrm{sP}$-selectin release than non-disabled subjects. Moreover, strenuous exercise, which enhanced release of prostacyclin and $\mathrm{NO}$ in healthy subjects, failed to so in those with SCI. Both prostacyclin and NO are potent anti-platelet agents [24]. Several mechanisms potentially explain why physical deconditioning/disability enhanced the extent of platelet reactivity. A previous study indicated that SCI patients were hypersensitive to platelet $\alpha_{2}$-adrenoreceptors [56]. That study showed that platelet aggregation by epinephrine was higher in SCI subjects than in healthy subjects [56]. Individuals with SCI may have decreased blood flow and even stasis resulting from lack of muscle pumping action in immobilized lower limbs. Early studies by Langille and O'Donnell demonstrated that chronically decreased blood flow caused a reduction in blood vessel diameter, which was likely mediated by low levels of endothelium-derived relaxing factors (EDRFs), such as prostacyclin and NO [57]. Additionally, previous investigations demonstrated that the reduced peripheral vascular resistance and the enhanced blood flow during exercise training attenuated after a deconditioning period, simultaneously accompanied by decreased plasma NO metabolite level [53, 58]. Therefore, we propose that either suppression of prostacyclin and NO productions or up-regulation of platelet $\alpha_{2}$-adrenoreceptor by physical deconditioning/disability promotes platelet reactivity and facilitates formation of hemostatic platelet plugging.

On the other hand, although resting and exercise-induced Ox-LDL-promoted platelet reactivity and plasma lipid peroxidation were decreased by exercise training [53, 54], this antioxidative effect of exercise training was reversed by deconditioning to the per-training state [55]. Additionally, individuals with SCI had more extensive resting and exercise-enhanced Ox-LDL-promoted platelet reactivity and greater lipid peroxides levels than those without SCI [24]. Therefore, supplemental therapy or intervention with anti-oxidants may be necessary for those with sedentary lifestyles and physical deconditioning, especially during strenuous exercise.

\section{Exercise and changes in coagulation and fibrinolysis}

\section{Effects of acute exercise}

Blood haemostasis is a complex interaction among platelets, coagulation, and fibrinolysis. According to the previous discussion, the intensity of acute exercise is a critical factor affecting blood platelet function [18, 22]; i.e., moderate exercise desensitizes platelets, whereas strenuous exercise potentiates platelets. The coagulatory and fibrinolytic responses to acute exercise may also be intensitydependant, illustrating that moderate exercise $\left(68 \% \mathrm{VO}_{2 \max }\right)$ enhances plasmin formation and fibrinolytic activity without correspondingly increasing markers of blood coagulation activation [59]. Strenuous exercise $\left(83 \% \quad \mathrm{VO}_{2 \max }\right)$, 
conversely, activates blood fibrinolysis and coagulation simultaneously [59]. Strenuous exerciseinduced hyper-coagulability as reflected by an increasing of FVIII concentration and shortening of activated partial thromboplastin time (APPT) $[60,61]$ suggests that this activation occurs though the intrinsic, but not the extrinsic coagulation pathway. The $\beta$-adrenergic pathway has been implicated as a pathway potentially mediating an exercise-induced increase in FVIII levels as $\beta$-blockade blunts this increase [62]. Some investigations showed that aerobic and anaerobic exercise increases plasmin formation, possibly by increasing plasminogen activator (t-PA) and reducing plasminogen activator inhibitor (PAI-1) $[34,63]$, thereby accelerating the blood fibrinolysis rate and enhancing fibrin degradation. Although $\beta$-adrenergic receptor blocking by propranolol partially reduces normal fibrinolytic response to strenuous exercise [64], t-PA release occurs prior to an increase in blood catecholamine concentration, suggesting that the initial release of t-PA induced by exercise is mediated by another non-adrenergic mechanism - possibly vasopressin [65].

\section{Effects of exercise training and physical deconditioning}

The effects of exercise training on blood coagulation and fibrinolysis have seldom been studied. A cross-sectional investigation showed no significant difference in prothrombin time (PT) and APPT at rest and post-exercise among sedentary subjects, joggers, and marathon runners [66]. Moreover, resting levels of FVIII activity and antigen in sedentary individuals [67] and endurance-trained athletes [68] do not change through training. However, a clinical investigation demonstrated that post-myocardial patients achieved lowered resting levels of FVIII activity and antigen after 4 weeks of physical training [7]. As data for the effects of exercise training on blood coagulation are incomplete and fragmented, further investigation is required.

Conversely, available cross-sectional data indicates that enhanced fibrinolysis in response to exercise is related to the training status of an individual: higher t-PA release and lower PAI-1 activity were identified in physically trained subjects than untrained subjects following exercise $[69,70]$. Measurement results in longitudinal investigations also demonstrated that young and elderly individuals had increased t-PA release and decreased PAI-1 activity and antigen following various training programs [67, 71]. However, 3 months of detraining tended to reverse favorable reductions in PAI activity observed post-training [72]. Therefore, exercise training reduces thrombi development and enhances blood clot degradation, likely by increasing plasmin formation and, subsequently, accelerating the rate of blood fibrinolysis.

\section{Conclusion}

Regulation of thrombogenesis is complex, involving various mechanisms, each of which can potentially be mediated by various regulatory factors or pathways. A number of thrombotic modifications have been reported in acute exercise, exercise training, and physical deconditioning/inactivity. Based on research to date, this study attempted to summarize the effects of acute exercise on platelets, coagulation and fibrinolysis as follows: light, acute exercise (i.e., $\leq 49 \% \mathrm{VO}_{2 \max }, \leq 59 \% \mathrm{HR}_{\max }, \leq 11$ on the RPE scale, $\leq 4$ MTEs) does not sufficiently

Table 2. Effects of exercise intensity on platelet function, coagulation, and fibrinolysis.

\begin{tabular}{llll}
\hline & Platelet function & Coagulation & Fibrinolysis \\
\hline Light exercise & $\mathrm{N}$ & $\mathrm{N}$ & $+^{\mathrm{a}}$ \\
Moderate exercise & $-{ }^{\mathrm{b}}$ & $\mathrm{N}$ & $+^{\mathrm{c}}$ \\
Strenuous exercise & $+^{\mathrm{d}}$ & $+^{\mathrm{e}}$ & $+^{\mathrm{c}}$ \\
\hline
\end{tabular}

+ , increase; - , decrease; $\mathrm{N}$, no change.

a $>50 \% \mathrm{HR}_{\max }[59]$.

${ }^{\mathrm{b}} 50-60 \% \mathrm{VO}_{2 \max }[18,22,31]$.

${ }^{\mathrm{c}} 70-90 \% W_{\max }[59]$.

d $>80 \% V_{2} V_{2 \max }[18,20-22,29]$.

e $>83 \% \mathrm{VO}_{2 \max }[61]$. 
influence platelet reactivity and coagulation, but increases fibrinolytic activity at intensity over $>50 \% \mathrm{HR}_{\max }$; moderate, acute exercise (i.e., 50 $74 \% \mathrm{VO}_{2 \max }, 60-79 \% \mathrm{HR}_{\max }, 12-13$ on the RPE scale, 5-6 METs) suppresses platelet reactivity and enhances fibrinolysis, but does not change coagulatory activity; and, strenuous, acute exercise (i.e., $\geq 75 \% \mathrm{VO}_{2 \max }, \geq 80 \% \mathrm{HR}_{\max }, \geq 14$ on the RPE scale, $\geq 7$ ) enhances platelet reactivity and coagulation, simultaneously promoting fibrinolytic activity (Table 2).

Abnormal hemostatic profiles are known to have clinical and prognostic relevance in cardiovascular disease. Enhanced platelet reactivity and coagulation in short-term vigorous exercise may accelerate the formation of hemostatic clot plugging, in turn causing thrombosis, and thus increasing the risk of cardiac arrest. Conversely, moderate-intensity exercise can plausibly be considered safe and effective exercise for minimizing thrombotic complications by eliciting anti-platelet and hyper-fibrinolytic effects. Moreover, longterm moderate exercise training (e.g., $60 \% \mathrm{VO}_{2 \max }$ for $30 \mathrm{~min}$ per day, 5 day/week for 8 weeks) reduces the prothrombotic state at rest and limits risk of thrombosis evoked by strenuous exercise. This phenomenon may explain, at least partially, why life habits with regular exercise protect individuals against cardiovascular disease and reduced this risk of cardiac arrest during vigorous exercise. Nevertheless, these favorable effects of exercise training on thrombotic modification reverse back to a pre-training state after a period of deconditioning and, hence, regular moderate exercise is critical maintaining its benefits.

\section{References}

1. Paffenbarger R.S., Hyde R.T., Wing A.L. et al., The association of changes in physical-activity level and other lifestyle characteristics with mortality among men. N. Engl. J. Med. 328: 538-545, 1993.

2. Blair S.N., Kohl H.W., Barlow C.E. et al., Changes in physical fitness and all-cause mortality: a prospective study of healthy and unhealthy men. JAMA 273: 1093-1098, 1995.

3. Pate R.R., Pratt M., Blair S.N. et al., Physical activity and public health: a recommendation from the Centers for Disease Control and Prevention and the American College of Sports Medicine. JAMA 273: 402-407, 1995.

4. Raven P.B. and Pawelczyk J.A., Chronic endurance exercise training: a condition of inadequate blood pressure regulation and reduced tolerance to LBNP. Med. Sci. Sports Exerc. 25: 713-721, 1993.
5. Lee K.W. and Lip G.Y.H., Effects of lifestyle on hemostasis, fibrinolysis, and platelet reactivity: a systematic review. Arch. Intern. Med. 163: 2368-2392, 2003.

6. Durstine J.L., Grandjean P.W., Davis P.G., Ferguson M.A., Alderson N.L. and DuBose K.D., Blood lipid and lipoprotein adaptations to exercise: a quantitative analysis. Sports Med. 31: 1033-1062, 2001

7. Womack C.J., Nagelkirk P.R. and Coughlin A.M., Exercise-induced changes in coagulation and fibrinolysis in healthy populations and patients with cardiovascular disease. Sports Med. 33: 795-807, 2003.

8. Green D.J., Maiorana A., O'Driscoll G. and Taylor R., Effect of exercise training on endothelium-derived nitric oxide function in humans. J. Physiol. 561( $\mathrm{Pt} 1)$ 1-25, 2004.

9. Albert C.M., Mittleman M.A., Chae C.U., Lee I.M., Hennekens C.H. and Manson J.E., Triggering of sudden death from cardiac causes by vigorous exertion. N. Engl. J. Med. 343: 1355-1361, 2000.

10. Siscovick D.S., Weiss N.S., Fletcher R.H. and Lasky T., The incidence of primary cardiac arrest during vigorous exercise. N. Engl. J. Med. 311: 984-987, 1984.

11. Scherer D. and Kaltenbach M., Frequency of life-threatening complications associated with exercise testing. Dtsch. Med. Wochenschr. 33: 1161-1165, 1979.

12. Cobb L.A. and Weaver W.D., Exercise: a risk for sudden death in patients with coronary heart disease. J. Am. Coll. Cardiol. 7: 215-219, 1986.

13. Mittleman M.A., Maclure M., Tofler G.H. et al., Triggering of acute MI by heavy physical exertion: protection against triggering by regular exertion. N. Engl. J. Med. 329: 1677-1683, 1993.

14. Willich S.N., Lewis M., Lowel H. et al., Physical exertion as a trigger of acute myocardial infraction. N. Engl. J. Med. 329: 1684-1690, 1993.

15. American College of Sports Medicine. General principles of exercise prescription. ACSM's guidelines for exercise testing and prescription. Seventh edition. Lippincott Williams \& Wilkins, 2006, pp. 133-173.

16. Fitzgerald D.J., Roy L., Catella F. and FitzGerald G.A., Platelet activation in unstable coronary disease. N. Engl. J. Med. 315: 983-989, 1986.

17. El-Sayed M.S., Ali N. and El-Sayed Ali Z., Aggregation and activation of blood platelets in exercise training. Sports Med. 35: 11-22, 2005.

18. Wang J.S., Jen C.J., Kung H.C., Lin L.J., Hsiue T.R. and Chen H.I., Different effects of strenuous exercise and moderate exercise on platelet function in men. Circulation 90: 2877-2885, 1994.

19. Wang J.S., Jen C.J. and Chen H.I., Effects of exercise training and deconditioning on platelet function in men. Arteriosc. Thromb. Vasc. Biol. 15: 1668-1674, 1995.

20. Wang J.S. and Cheng L.-J., The effect of strenuous acute exercise on $\alpha_{2}$-adrenergic agonist-potentiated platelet activation. Arteriosclero. Thromb. Vasc. Biol. 19: 1559-1565, 1999.

21. Wang J.S., Chow S.E. and Chen J.-K., Strenuous, acute exercise affects reciprocal modulation of platelet and polymorphonuclear leukocyte activities under shear flow in men. J. Thromb. Haemost. 1: 2031-2037, 2003.

22. Wang J.S., Jen C.J., Lee H. and Chen H.I., Effects of shortterm exercise on female platelet function during different phases of the menstrual cycle. Arteriosclero. Thromb. Vasc. Biol. 17: 1682-1686, 1997. 
23. Wang J.S., Jen C.J. and Chen H.I., Effects of chronic exercise and deconditioning on platelet function in women. J. Appl. Physiol. 83: 2080-2085, 1997.

24. Wang J.S., Yang C.F., Wong M.-K., Chow S.-E. and Chen J.-K., Effect of strenuous arm exercise on oxidized-LDLpotentiated platelet activation in individuals with spinal cord injury. Thromb. Haemost. 84: 118-123, 2000.

25. Wang J.S., Yang C.F. and Wong M.-K., Effect of strenuous, arm exercise on platelet function in patients with spinal cord injury. Arch. Phys. Med. Rehabil. 83: 210216, 2002

26. Kestin A.S., Ellis P.A., Barnard M.R., Errchetti A., Rosner B.A. and Michelson A.D., Effect of strenuous exercise on platelet activation state and reactivity. Circulation 88(Part 1) $1502-1511,1993$.

27. Holme P.A., Orvim U., Hamers M.J., Solum N.O., Brosstad F.R., Barstad R.M. and Sakariassen K.S., Shear-induced platelet activation and platelet microparticle formation at blood flow conditions as in arteries with a severe stenosis. Arterioscler. Thromb. Vasc. Biol. 17: 646653, 1997.

28. Strony J., Beaudoin A., Brands D. and Adelman B., Analysis of shear stress and hemodynamic factors in a model of coronary artery stenosis and thrombosis. Am. J. Physiol. 265: H1787-H1796, 1993.

29. Wang J.S., Intense exercise increases shear-induced platelet aggregation through enhancement of von Willebrand factor Binding, glycoprotein IIb/IIIa activation and P-selectin expression. Eur. J. Appl. Physiol. 91: 741-747, 2004.

30. Tokuue J., Hayashi J., Hata Y., Nakahara K. and Ikeda Y., Enhanced platelet aggregability under high shear stress after treadmill exercise in patients with effort angina. Thromb. Haemost. 75: 833-837, 1996.

31. Wang J.S. and Liao C.H., Moderate-intensity exercise suppresses platelet activation and polymorphonuclear leukocytes interaction with surface-adherent platelets under shear flow in men. Thromb. Haemost. 91: 587594, 2004.

32. Kaplan K.L. and Owen J., Plasma levels of $\beta$-thromboglobulin and platelet factor 4 as indices of platelet activation in vivo. Blood 57: 199-202, 1981.

33. Ahmadizad S. and El-Sayed M., The effect of graded resistance exercise on platelet aggregation and activation. Med. Sci. Sports Exerc. 35: 1026-1032, 2003.

34. El-Sayed M.S., El-Sayed Ali Z. and Ahmadizad S., Exercise and training effects on blood haemostasis in health and disease: an update. Sport Med. 34: 181-200, 2004.

35. Mazzeo R.S., Colburn R.W. and Horvath S.M., Effect of aging and endurance training on tissue catecholamine response to strenuous exercise in Fischer 344 rats. Meta Clin. Exp. 35: 602-607, 1986

36. Vischer U.M. and Wollheim C.B., Epinephrine induces von Willebrand factor release from cultured endothelial cells: involvement of cyclic AMP-dependent signalling in exocytosis. Thromb. Haemost. 77: 1182-1188, 1997.

37. Goto S., Ikeda Y., Murata M. et al., Epinephrine augments von Willebrand factor-dependent shear-induced platelet aggregation. Circulation 86: 1859-1863, 1997.

38. Wagner C.T., Kroll M.H., Chow T.W., Hellums J.D. and Schafer A.I., Epinephrine and shear stress synergistically induce platelet aggregation via a mechanism that partially bypasses VWF-GP Ib interactions. Biorheology 33: 209-229, 1996.
39. Kaul S., Makkar R.R., Nakamura M. et al., Inhibition of acute stent thrombosis under high-shear flow conditions by a nitric oxide donor, DMHD/NO. An ex vivo porcine arteriovenous shunt study. Circulation 94: 2228-2232, 1996.

40. Michelson A.D., Benoit S.E., Furman M.I. et al., Effects of nitric oxide/EDRF on platelet surface glycoproteins. Am. J. Physiol. 270: H1640-1648, 1996.

41. World Health Statistics Annual. Vital statistics and causes of death 1980. Geneva, WHO 1980.

42. Diacovo T.G., Roth S.J., Buccola J.M., Bainton D.F. and Springer T.A., Neutrophil rolling, arrest, and transmigration across activated, surface-adherent platelets via sequential action of P-selectin and the $\beta 2$-integrin CD11b/CD18. Blood 88: 146-157, 1996.

43. Hagberg I.A., Roald H.E. and Lyberg T., Adhesion of leukocytes to growing arterial thrombosis. Thromb. Haemost. 80: 852-858, 1998.

44. Faint R.W., Mackie I.J. and Machin S.J., Platelet aggregation is inhibited by a nitric oxide-like factor released from human neutrophils in vitro. Br. J. Haematol. 71: 539-545, 1991.

45. Zatta A., Prosdocimi M., Bazzoni G., Dejana E. and Del Maschio A., Inhibition of platelet function by polymorphonuclear leukocytes. J. Lab. Clin. Med. 116: 651-660, 1990.

46. Wong C.S., Gamble J.R., Skinner M.P. et al., Adhesion protein GMP 140 inhibits superoxide anion release by human neutrophils. Proc. Natl. Acad. Sci. USA 88: 23972401, 1991.

47. Li N., Wallen N.H. and Hjemdahl P., Evidence for prothrombotic effects of exercise and limited protection by aspirin. Circulation 100: 1374-1379, 1999.

48. Yamamoto J., Lizumi H., Hirota R., Shimonaka K., Nagamatsu Y., Horie N. and Morita S., Effect of physical training on thrombotic tendency in rats: decrease in thrombotic tendency measured by the He-Ne laser-induced thrombus formation method. Haemostasis 19: 260-265, 1989.

49. Wang J.S., Li Y.-S. and Chen J.-C., Effects of exercise training and deconditioning on platelet Aggregation induced by alternating shear stress in men. Arteriosclero. Thromb. Vasc. Biol. 25: 454-460, 2005.

50. Mazzeo R.S., Catecholamine responses to acute and chronic exercise. Med. Sci. Sports Exerc. 23: 839-845, 1991.

51. Lehmann M., Hasler K., Bergdolt E. and Keul J., Alpha2-adrenoreceptor density on intact platelets and adrenaline-induced platelet aggregation in endurance-and nonendurance-trained subjects. Int. J. Sports Med. 7: 172-176, 1986.

52. Shern-Brewer R., Santanam N., Wetzstein C., WhiteWelkley J. and Parthasarathy S., Exercise and cardiovascular disease: a new perspective. Arterioscler. Thromb. Vasc. Biol. 18: 1181-1187, 1998.

53. Wang J.S., Lin C.-C., Chen J.-K. and Wong M.-K., Role of chronic exercise in decreasing oxidized LDL-potentiated platelet activation by enhancing platelet-derived NO release and bioactivity in rats. Life Sci. 66: 1937-1948, 2000.

54. Wang J.S., Chow S.-E., Chen J.-K. and Wong M.-K., Effect of exercise training on oxidized LDL-mediated platelet function in rats. Thromb. Haemost. 83: 503-508, 2000.

55. Wang J.S. and Chow Shu-Er, Effects of exercise training and detraining on oxidized LDL-mediated platelet 
function in men. Arch. Phys. Med. Rehabil. 85: 15311537, 2004.

56. Senard J.M., Arias A., Berlan M., Tran M.A., Rascol A. and Montastruc J.L., Pharmacological evidence of alpha 1and alpha 2-adrenergic supersensitivity in orthostatic hypotension due to spinal cord injury: a case report. Eur. J. Clin. Phyarmacol. 41: 593-596, 1991.

57. Langile B.L. and O'Donnell F., Reductions in arterial diameter produced by chronic decreases in blood flow are endothelium-dependent. Science 231: 405-407, 1988.

58. Wang J.S., Effects of exercise training and detraining on cutaneous microvascular function in Men: the regulatory role of endothelium-dependent dilation in skin vasculature. Eur. J. Appl. Physiol. 93: 429-434, 2005.

59. Weiss C., Seitel G. and Bartsch P., Coagulation and fibrinolysis after moderate and very heavy exercise in healthy male subjects. Med. Sci. Sports Med. 30: 246-251, 1998.

60. El-Sayed M.S., Effects of exercise on blood coagulation, fibrinolysis and platelet aggregation. Sports Med. 22: 282298, 1996.

61. El-Sayed M.S., Sale C., Jones P. et al., Blood haemostasis in exercise and training. Med. Sci. Sports Exerc. 32: 918925, 2000.

62. Cohen R.J., Epstein S.E., Cohen L.S. et al., Alterations in blood fibrinolysis and blood coagulation induced by exercise and the role of beta-adrenergic receptor stimulation. Lancet II: 1264-1266, 1968.

63. Andrew M., Carter C., O'Brodovich H. et al., Increases in factor VIII complex and fibrinolytic activity are dependent on exercise intensity. J. Appl. Physiol. 60: 1917-1922, 1986.
64. El-Sayed M.S. and Davies B.A., Effect of two formulations of beta-blocker on fibrinolytic response to maximum exercise. Med. Sci. Exerc. 21: 369-373, 1989.

65. El-Sayed M.S., Exercise intensity-related response of fibrinolytic activity and vasopressin in man. Med. Sci. Sports Exerc. 22: 494-500, 1990.

66. Ferguson E.W., Bernier L.L., Banata G.R. et al., The effect of exercise and conditioning on clotting and fibrinolytic activity in men. J. Appl. Physiol. 62: 1416-1421, 1987.

67. Vanden Burg P.J.M., Hospers J.E., van Vliet M. et al., Effect of endurance training and seasonal fluctuation on coagulation and fibrinolysis in young sedentary men. $\mathbf{J}$. Appl. Physiol. 82: 613-620, 1997.

68. Watts E.J., Haemostatic changes in long-distance runners and their relevance to the prevention of ischemic heart disease. Blood Coagul. Fibrinolysis 2: 221-225, 1991.

69. Szymanski L.M. and Pate R.R., Effect of exercise intensity, duration, and time of day on fibrinolytic activity in physically active men. Med. Sci. Sports Exerc. 26: 11021108, 1994.

70. Szymanski L.M., Pate R.R. and Durstine J.L., Effects of maximal exercise and venous occlusion on fibrinolytic activity in physically active and inactive men. J. Appl. Physiol. 77: 2305-2310, 1994.

71. Stratton J.R., Chandler W.L., Schwartz R.S. et al., Effects of physical conditioning on fibrinolytic variables and fibrinogen in young and old healthy adults. Circulation 83: 1692-1697, 1991.

72. Gris J.C., Schved J.F., Feugeas O. et al., Impact of smoking, physical training and weight reduction on FVIII, PAI-1 and hemostatic markers in sedentary men. Thromb. Res. 64: 516-520, 1990. 